\title{
Review of: "Molecular characterization of multidrug resistant Enterobacterales strains isolated from liver and kidney transplant recipients in Spain"
}

Cindy Shuan Ju Teh ${ }^{1}$

1 Universiti Malaya

Potential competing interests: The author(s) declared that no potential competing interests exist.

This is a very good paper describing the antimicrobial susceptibility and the resistance mechanisms of Enterobacteriaceae isolates from patients who have undergone liver, kidney or pancreas transplantation in Spain. 7 hospitals were involved in this study from August 2014 to April 2018 and 931 patients were recruited. However, only 308 patients contributed isolates of pre and/or post transplantation and only isolates $(n=531)$ from 247 patients were included in further analysis. Among the 247 patients, 10 developed infection.

All the isolates were subjected to antimicrobial testing for 24 agents and REP-PCR. Based on REP-profiling, 287 were further analysed with PFGE. Detectiion of ESBL, carbapenemase and AmpC genes were detected from 345 MDRE. Among 345 MDRE isolates, majority of the strains harbour ESBL genes. Carbapenemase genes (Oxa48, VIM, KPC2) were detected in 73 isolates which showed meropenem MIC>0.125mg/L. This study provided useful data for epidemiology and patient management. However, this paper could be further improved:

1. Information is needed for clarification of the flow and number of isolates included for analysis.

2. Which hospitals did not submit the strains? From which hospitals the 247 patients came from?

3. REP-PCR result was not reported in the manuscript.

4. All the isolates $(n=541)$ were included for MIC, but 345 were subjected to resistance genes detection. What about the other 196 isolates?

5. It would be good if the author could indicate the rates of infection post-transplantation in the introduction and provide information on the number and type of transplant surgery in each hospital for determination of the risk.

6. The clinical data of the 10 infection cases should be described and correlated with the phenotypic and genotypic characteristics. What is the clinical outcome?

7. More discussion is needed for the correlation of antimicrobial susceptibility profile and resistance mechanism.

8. The number of isolates were high for 1st month post-transplantation. Why? any previous reports? 
